\title{
Cytogenetic and Hematological Effects of the Antibiotic Chloramphenicol on Calves
}

\author{
Othman E. Othman ${ }^{1, *}$, Galila A. El-Rafey ${ }^{2}$ and Tarek A. Hassan ${ }^{2}$ \\ ${ }^{1}$ Cell Biology Department, National Research Center, Dokki, Giza, Egypt \\ ${ }^{2}$ Animal Reproduction Research Institute, Agriculture Research Center, Giza, Egypt
}

Received November 15, 2004; accepted January 20, 2005

\begin{abstract}
Summary The present study demonstrated the cytogenetic and hematological effects of the antibiotic chloramphenicol in blood cultures of calves, at 4 days and 3 weeks after stoppage of injection with chloramphenicol by the minimal therapeutic dose (once a day for 4 successive days).

The different types of chromosomal aberrations including breaks, gaps, deletions and fragments were increased in blood cultures after injection with chloramphenicol at significant levels ranging from $p<0.05$ to $p<0.01$. The total number of structural chromosomal aberrations was increased at highly significant level $p<0.001$ when compared with control.

The frequencies of sister chromatid exchanges in blood cultures of calves after the injection with chloramphenicol were significantly increased, whereas the mitotic index was decreased at significant levels ranging from $p<0.01$ to $p<0.001$.

The hematological analysis showed that there is no change in hemoglobin concentration after injection with chloramphenicol. Leucocytosis was shown only at 4 days after the stoppage of injection with chloramphenicol, whereas the lymphopenia and the neutrophilia were shown at 21 days after the stoppage of injection.
\end{abstract}

Key words Chloramphenicol, Chromosomal aberrations, Mitotic index, SCEs, Hematology.

Chloramphenicol (CAP) is a highly active broad-spectrum antibiotic with excellent pharmacokinetic properties. It has a broad spectrum of activity and good penetration of cells, most tissues and secretions. CAP is a non-ionized, highly lipid-soluble molecule that crosses cellular barriers to be widely distributed in body tissues and fluids (Barragry 1994).

Biologically, CAP can cause 2 types of toxic manifestations in bone marrow. The more commonly encountered one is bone marrow depression, which is reversible, and dose related and which occurs concurrently during administration of the antibiotic. The other one is the bone marrow aplasia, which is irreversible and often fatal and which occurs sometimes (often weeks and months) after administration of the antibiotic has been discontinued (El-Mofty et al. 2000). Either of them can occur in humans, whereas only bone marrow suppression has been reported in domestic animals (Barragry 1994, Plumb 1999, Turton et al. 2002).

The most prevalent toxic effects observed after administration of CAP in the eukaryotic cells are explicable in terms of the demonstrated inhibitions of mitochondrial function and protein synthesis. Because it is a protein-synthesis inhibitor, CAP can interfere with synthesis of immunoglobulin and multiplying cells are the most adversely affected. This would account for the exhaustion and aplasia seen in humans and animals after administration of CAP (Summ et al. 1976).

The purpose of the present study is to evaluate the cytogenetic and hematological effects of CAP in calves. The formation of chromosomal aberrations, the induction of sister chromatid exchanges and the mitotic index are the 3 cytogenetic parameters used in this study. The hematolog-

\footnotetext{
* Corresponding author, e-mail: othmanmah@yahoo.com
} 
ical analysis included the measurement of hemoglobin concentration, total leucocytic count and differential leucocytic count.

Materials and methods

Drug

CAP was first isolated from cultures of Streptomyces venezuelae in 1947 and was chemically synthesized in 1949.

Chloramphenicol succinate is highly soluble in water and is hydrolyzed in tissues with the liberation of free CAP; it is used for parenteral injection.

\section{Blood samples and culture of lymphocytes}

Eight female calves (2-4 months old) were assigned for this work. The animals were daily intramuscularly injected with CAP sodium citrate $(10 \mathrm{mg} / \mathrm{kg}$, bw) once a day for 4 successive days. This is the minimal therapeutic dose restricted for which benefits of the drug outweigh the risks of the potential toxicities (Gilman 1992). Three blood samples were collected from each animal, the first one just before injection (as control), the second at 4 days after stoppage of injection and the last one at 3 weeks after stoppage of injection.

For cytogenetic study, $1 \mathrm{ml}$ of whole heparinized blood was cultured at $38.5^{\circ} \mathrm{C}$ for $72 \mathrm{~h}$ in $5 \mathrm{ml}$ RPMI 1640 medium (Gibco) supplemented with 20\% fetal calf serum (Gibco), 0.1\% penicillinstreptomycin (Gibco), 1\% L-glutamine (Gibco) and 4\% phytohaemaglutinin (Gibco). The blood cultures for sister chromatid exchanges (SCEs) were treated with bromodeoxyuridine (BrdU, Sigma) at a final concentration of $10 \mu \mathrm{g} / \mathrm{ml}, 24 \mathrm{~h}$ after culture initiation.

\section{Chromosome preparation and staining}

$11 / 2 \mathrm{~h}$ before harvesting, colchicine was added to the cultures at a final concentration of $20 \mu \mathrm{g} / \mathrm{ml}$. At harvest, the cells were treated with a hypotonic solution $(0.075 \mathrm{M} \mathrm{KCl})$ and incubated at $37^{\circ} \mathrm{C}$ for $20 \mathrm{~min}$. Then the cells were fixed 3 times with fixative ( 3 methanol: 1 acetic acid). Finally, the cells were spread onto cold slides, dipped in $70 \%$ ethyl alcohol and the slides were air dried for subsequent different staining techniques.

For chromosomal aberrations and mitotic index, the slides were stained with $10 \%$ Giemsa stain solution (diluted with phosphate buffer, $\mathrm{pH} \mathrm{6.8)} \mathrm{for} 30 \mathrm{~min}$ and washed twice in phosphate buffer. The slides were air dried and examined under light microscope.

For sister chromatid exchanges, the fluorescence-photolysis-giemsa technique (Latt et al. 1981) was used. The slides were stained with an aqueous solution of Hoechst $33258(50 \mu \mathrm{g} / \mathrm{ml}$, Sigma) for $20 \mathrm{~min}$ and then rinsed in distilled water. On a warmer tray at $50^{\circ} \mathrm{C}$, the slides were layered with McIlvaines buffer and subjected to fluorescent black blue light at a distance of $5 \mathrm{~cm}$ for $50 \mathrm{~min}$. The slides were stained with $4 \%$ Giemsa stain solution ( $\mathrm{pH} 7)$. The slides were rinsed in distilled water and examined under light microscope.

\section{Scoring and statistical analysis}

For each animal, 50 well-spread metaphases were examined for chromosomal aberrations, whereas 30 metaphases in the second division were analyzed for SCEs. One thousands cells/animal were examined for measuring the mitotic index.

A $\chi^{2}$ test $(2 \times 2$ contingency table) was used for the chromosomal aberrations data analysis, while the $t$-test was used to evaluate sister chromatid exchanges and mitotic index.

\section{Hematological analysis}

For hematological study, blood samples were collected on EDTA for measurement of hemo- 
globin concentration ( $\mathrm{Hb}$ ) by using Drabkin's solution, total leucocytic count (TLC) by using Turk's solution and improved Neubouer hemocytometer and differential leucocytic count by using stainedblood films with Leishman's stain according to Schalm (1986). One hundred cells on the edge of the smear were differentiated and the percentage of different leucocytes was calculated. Data were collected and statistically analyzed by using a computer statistical system PC STAT.

\section{Results}

\section{Cytogenetic effect of CAP}

\section{Analysis of chromosomal aberrations}

The frequency of structural chromosomal aberrations was recorded in blood cultures of calves before the treatment with CAP and at 4 days as well as 3 weeks after the stoppage of the treatment (Table 1). The normal metaphase without any chromosomal aberrations is shown in Fig. 1. The chromosomal aberrations screened in this study involved breaks (Fig. 2), gaps (Fig. 3) and deletions and fragments (Figs. 4, 5).

After 4 days from the last day of injection with CAP, the numbers of cells with breaks and gaps were increased at significant level $p<0.01$ when compared with controls, whereas the number of cells with deletions and fragments was significantly increased at $p<0.05$. The results showed that the number of total aberrant cells with structural chromosomal aberrations was increased at highly significant level $p<0.001$ compared with controls.

After 3 weeks from the last day of injection, the numbers of cells with breaks and gaps were increased at significant level $p<0.05$. The number of cells with deletions and fragments was also increased but the increase not reach to any significant level when compared with controls. The results also showed that the number of total aberrant cells with structural chromosomal aberrations was increased at highly significant level $p<0.001$.

\section{Sister chromatid exchanges}

The effect of CAP on the frequency of sister chromatid exchanges was shown in Table 2. For each animal, thirty metaphases in second division were examined for SCEs before treatment and 4 days as well as 3 weeks after the last day of injection. The means of SCEs/cell \pm SD after 4 days and 3 weeks were $9.81 \pm 1.83$ and $7.68 \pm 1.35$, respectively compared to $5.53 \pm 0.92$ in the cultures before treatment. By using the $t$-test for the analysis of SCEs data, we found that CAP increased the frequency of sister chromatid exchanges in cultures after 4 days from last injection at highly significant level $(p<0.001)$, whereas it increased SCEs in cultures after 3 weeks at a significant level, $p<0.01$. Fig. 6 shows a metaphase with few SCEs before treatment, whereas Fig. 7 shows a metaphase with many SCEs after treatment with CAP.

Table 1. Frequency of chromosomal aberrations induced in calves after treatment with chloramphenicol

\begin{tabular}{|c|c|c|c|c|c|c|c|c|c|c|}
\hline \multirow{3}{*}{$\begin{array}{c}\text { Drug } \\
\text { treatment }\end{array}$} & \multirow{3}{*}{$\begin{array}{c}\text { No. of } \\
\text { animals }\end{array}$} & \multirow{3}{*}{$\begin{array}{c}\text { No. of } \\
\text { examined } \\
\text { cells }\end{array}$} & \multicolumn{6}{|c|}{ No. of different chromosomal aberrations } & \multirow{2}{*}{\multicolumn{2}{|c|}{$\begin{array}{c}\text { Total number of } \\
\text { chromosomal } \\
\text { aberrations }\end{array}$}} \\
\hline & & & \multicolumn{2}{|c|}{ Breaks } & \multicolumn{2}{|c|}{ Gap } & \multicolumn{2}{|c|}{$\begin{array}{l}\text { Deletions, } \\
\text { fragments }\end{array}$} & & \\
\hline & & & No. & $\%$ & No. & $\%$ & No. & $\%$ & No. & $\%$ \\
\hline Before treatment & 8 & 400 & 15 & 3.75 & 18 & 4.5 & 10 & 2.5 & 43 & 10.75 \\
\hline After 4 days & 8 & 400 & $33 * *$ & 8.25 & $39 * *$ & 9.75 & $21^{*}$ & 5.25 & $93 * * *$ & 23.25 \\
\hline After 3 weeks & 8 & 400 & $31 *$ & 7.75 & $34 *$ & 8.5 & 17 & 4.25 & $82 * * *$ & 20.5 \\
\hline
\end{tabular}

$* p<0.05, * * p<0.01, * * * p<0.001$. 

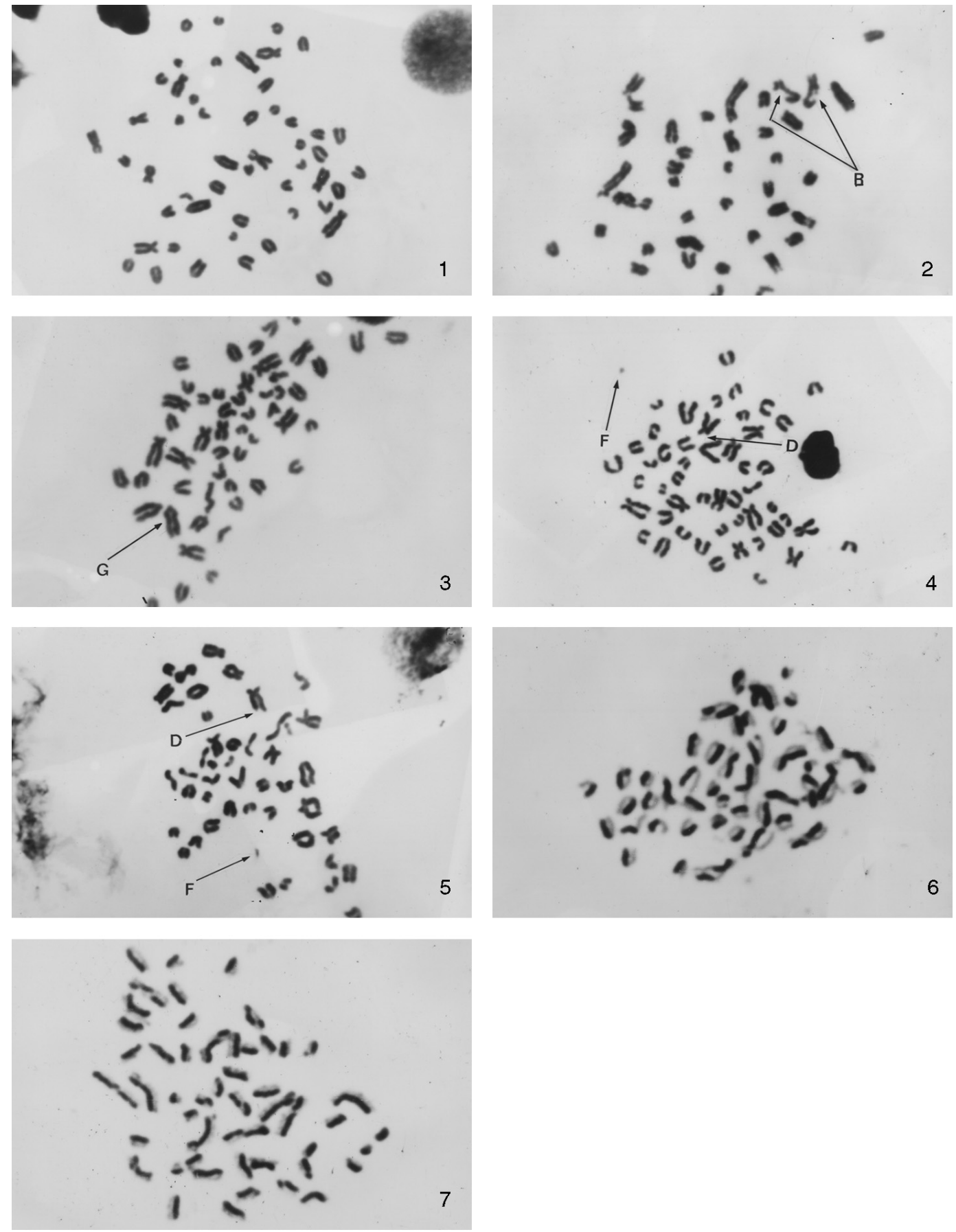

Figs. 1-7. 1. Metaphase spread of calve lymphocyte showing normal calve chromosomes. 2. Metaphase spread of calve lymphocyte showing 2 chromatid breaks. 3. Metaphase spread of calve lymphocyte showing a chromatid gap. 4. Metaphase spread of calve lymphocyte showing a deletion in the short arm of chromosome and a fragment. 5. Metaphase spread of calve lymphocyte showing a deletion in the long arm of chromosome and a fragment. 6. Metaphase spread of calve lymphocyte showing few sister chromatid exchanges. 7. Metaphase spread of calve lymphocyte showing many sister chromatid exchanges. 

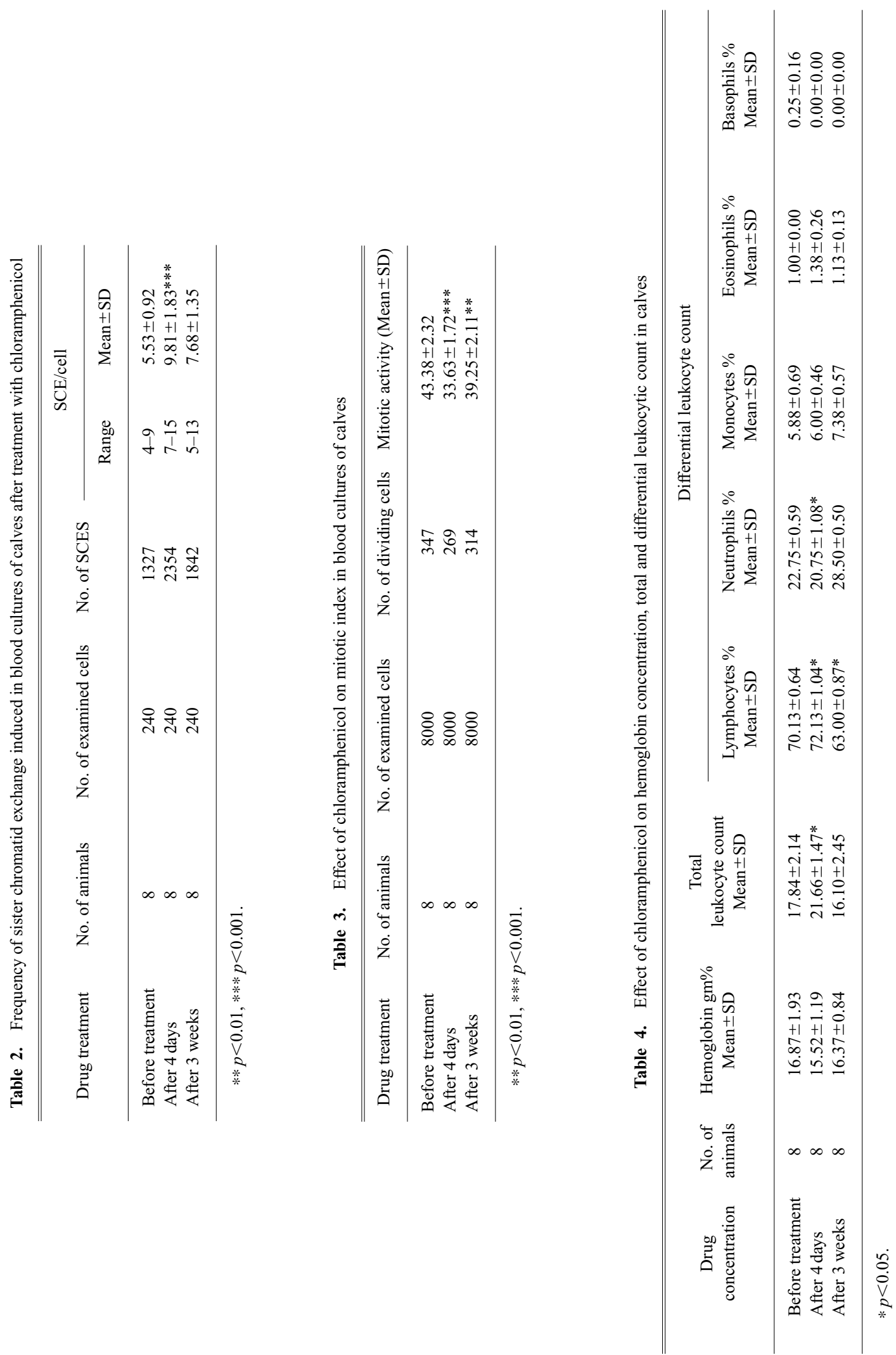


\section{Mitotic index}

The effect of CAP on mitotic index in blood cultures of calves was studied in this work (Table $3)$. The results showed that CAP decreased the mitotic index $(33.63 \pm 1.72)$ at a highly significant level $(p<0.001)$ in blood cultures after 4 days from the last injection. After 3 weeks from treatment with CAP, the mitotic index was decreased (39.25 \pm 2.11$)$ at significant level $p<0.01$ when compared with controls $(43.38 \pm 2.32)$.

\section{Hematological effect of CAP}

Hematological changes that occurred in blood of treated calves were shown in Table 4. There was no effect of CAP on the mean values of hemoglobin concentration after 4 days as well as after 21 days in comparison to that before treatment. Total leucocytic count showed a significant increase at $p<0.05$ (leukocytosis) at 4 days after the stoppage of injection, but had a non-significant decrease (leucopenia) at 21 days after the stoppage of injection. Regarding to the differential leucocytic count, there was non-significant lymphocytosis at 4 days whereas there was a relative significant lymphopenia $(p<0.05)$ at 21 days after the stoppage of injection. On the other hand, there was nonsignificant decrease in neutrophils count at 4 days after the stoppage of injection and their number was increased significantly at $p<0.05$ after 21 days. Regarding the monocytes, eosinophils and basophiles, there were no changes in their counts either at 4 or at 21 days after the stoppage of CAP injection.

\section{Discussion}

CAP, a highly active, broad-spectrum antibiotic with excellent pharmacokinetic properties, is one of the most discussed drugs. It has a broad spectrum of antibacterial activity, which includes both Gram-positive and Gram-negative organisms. CAP is active against all types of anaerobes and also against rickettsial and chlamydial species, but not mycoplasmas (Wilson and Cockerill 1983).

CAP can cause 2 types of toxic manifestations in bone marrow. The more common one is bone marrow depression which is reversible, and the other is the bone marrow aplasia which is irreversible and often fatal (Lubran 1989, El-Mofty et al. 2000). Either of them can occur in humans, whereas only bone marrow suppression has been reported in domestic animals (Turton et al. 2002). The most prevalent toxic effects observed after administration of CAP are explicable in terms of the demonstrated inhibitions of mitochondria's function and protein synthesis.

The cytogenetic effect of CAP was tested in this study. The different types of structural chromosomal aberrations induced in blood cultures of calves after treatment with CAP declared that the mutagenic effect of this drug was permanent aftet 3 weeks from the last injection. The frequencies of breaks $(7.75 \%)$, gaps $(8.5 \%)$ and deletions and fragments $(4.25 \%)$ after 3 weeks from the last injection was decreased slightly than those after 4 days, where the breaks $(8.25 \%)$, gaps $(9.75 \%)$ and deletions and fragments (5.25\%). But these structural aberrations still highly significantly increased over the controls (Table 1). The total numbers of structural chromosomal aberrations in blood cultures after 4 days from the last injection (23.25\%) as well as in cultures after 3 weeks $(20.5 \%)$ were increased at highly significant level $p<0.001$ when compared to the controls $(10.75 \%)$.

This mutagenic effect of CAP on chromosomes was reported by Goh (1979). Eight-hundred $\mu \mathrm{g}$ of CAP was added to $10 \mathrm{ml}$ of normal phytohemagglutinin-stimulated leukocyte cultures at various stages of cell cycle. More abnormalities were seen in the CAP added cultures. The highest incidence was seen when CAP was added at $G_{0}$ and the lowest at $G_{2}$. It was concluded that CAP is capable of inhibiting protein synthesis resulting in chromosomal protein deficiency. This deficiency produces "weakness" in the chromosomal backbone.

Skolimowski et al. (1983) studied the effect of CAP on DNA in vitro. They found that CAP caused DNA damage which was characterized by helix destabilization and strand breakage. 
The mutagenic effect of CAP in rats was studied by Joshi et al. (1987). The study revealed the incidence of both numerical and structural aberrations in somatic chromosomes after administration of CAP. These aberrations included chromatid gaps, chromatid breaks, fragments, centric fusions, dicentric chromosomes and polyploidy.

Sbrana et al. (1991) studied the induction of chromosomal aberrations in human lymphocyte cultures and in bone marrow cells of mice and in Chinese hamster cell cultures (V79) treated with CAP. While no aberrations were induced by short time of treatments in human lymphocytes exposed in $G_{1}$ and $G_{2}$ phases, high frequencies of aberrations, exclusively of the chromatid type, were induced when the drug was administered during a whole cell cycle. In mouse bone marrow cells, CAP induced a high mitotic delay and structural chromosomal aberrations.

The ability of CAP and its metabolites to induce DNA damage in human peripheral blood lymphocytes were studied by Lafarge-Frayssinet et al. (1994) and Robbana-Barnat et al. (1997). They reported the induction of DNA single-strand breaks and this induction of aberrations was dose-dependent.

The other parameter used in this study to evaluate the mutagenic effect of CAP is the induction of sister chromatid exchanges. The result showed that CAP increased the frequency of SCEs in cultures after 4 days from last injection at highly significant level $(p<0.001)$, whereas it increased SCEs in cultures after 3 weeks at a significant level, $p<0.01$.

This mutagenic effect of CAP on SCEs agreed with the results obtained by Sbrana et al. (1991). They reported that this antibiotic increased the SCES frequency in human lymphocyte cultures as well as in Chinese hamster cell cultures (V79).

The effect of CAP on SCEs in bovine was studied by Arruga et al. (1992) and Catalan et al. (1993). The former study showed the increased frequency of SCEs in bovine fibroblasts after treatment with different CAP concentrations. The same mutagenic effect of this antibiotic on SCEs frequency in bovine lymphocyte cultures was reported by Catalan et al. (1993).

In this study, the injection of CAP $(10 \mathrm{mg} / \mathrm{kg} \mathrm{bw})$ once daily for 4 days had no effect on the hemoglobin concentration. This result agreed with the studies of Burrows et al. $(1984,1988)$.

Regarding to the leucogram, this study revealed leukocytosis at 4 days after stoppage of the injection. This might be as a response of the animal where the intramuscular injection acts as a stress (Hassan 1995). In this study, CAP caused leucopenia at 21 days after stoppage of administration for its toxic effect and this result is in agreement with Meissner and Smith (1979), Watson (1980) and Nashwa (2003). Leucopenia after 21 days occurred due to the inhibition of leucocyte migration through the capillary endothelial lining of blood vessels (Jain 2000). CAP also altered neutrophils morphology and function (Paape et al. 1990).

Significant neutropenia was occurred at 4 days after stoppage of injection, this may be due to bone marrow suppression as have been mentioned by Paape et al. (1990) and Nashwa (2003). Also, marked lymphopenia after 21 days from injection was recorded in this study, which might be occurred due to dissolution of lymphocyte after exogenous or endogenous excess of gluco-corticoid as a response to intramuscular injection of CAP (Coles 1986).

The hematological changes in calves injected by CAP are similar to the reversible dose-related changes seen commonly during CAP therapy. The drastic effects of CAP seem to take longer time due to the reduced capability of calves to eliminate CAP efficiency (Burrows et al. 1984).

As a conclusion, the cytogenetic and hematological studies emphasize that CAP has marked mutagenic and toxic effects and that the calves should be given CAP only at minimum dose required to produce satisfactory antibiotic effects and in situations in which it is necessary. This drug should never be used in treatment of diseases that are readily, safely and affectively treatable with other antibiotic agents. 


\section{References}

Arruga, M. V., Catalan, J. and Moreno, C. 1992. Effect of chloramphenicol on sister chromatid exchange in bovine fibroblasts. Res. Vet. Sci. 52: 256-259.

Barragry, T. B. 1994. Veterinary Drug Therapy. Lea \& Febiger, Philadelphia. pp. 275-281.

Burrows, G. E., Tyler, R. D., Cralgmill, A. L. and Bartop, B. 1984. Chloramphenicol and the neonatal calf. Am. J. Vet. Res. 4: $1586-1591$.

-, 一, San Ggkia, S. and Keeton, R. D. 1988. Experimental chloramphenicol intoxication in neonatal Calves: Intravenous administration. Res. Vet. Sci. 45: 101-106.

Catalan, J., Moreno, C. and Arruga, M. V. 1993. Sister-chromatid exchanges induced by chloramphenicol on bovine lymphocytes. Mutat. Res. 319: 11-18.

Coles, E. H. 1986. Veterinary Clinical Pathology, 4th ed. W.B. Sounders, Philadelphia.

El-Mofty, M. M., Abdelmeguid, N. E., Sadek, I. A., Essawy, A. E. and Aleem, E. A. 2000. Induction of leukemia in chloramphenicol-treated toads. East Mediterr. Health J. 6: 1026-1034.

Gilman, A. G. 1992. The Pharmacological Basis of Therapeutics, 8th ed., Vol. 2, Chap. 48. McGraw-Hill, New York.

Goh, K. 1979. Chloramphenicol and chromosomal morphology. J. Med. 10: 159-166.

Hassan, A. T. 1995. Haematological and endocrinological studies on stress animals with special reference to their reproductive efficiency. Ph.D. thesis, Fsc. Vet. Med., Cairo Univ.

Jain, N. C. 2000. Schalm's Veterinary Haematology, 5th ed. Lea \& Febiger, Philadelphia.

Joshi, S. K., Katpatal, B. G. and Sarkhel, B. C. 1987. Effect of chloramphenicol on somatic chromosomes of rats. Ind. Vet. Med. J. 11: 129-134.

Lafarge-Frayssinet, C., Robbana-Barnat, S., Frayssinet, C., Toucas, L. and Decloitre, F. 1994. Cytotoxicity and DNA damaging potency of chloramphenicol and six metabolites: A new evaluation in human lymphocytes and Raji cells. Mut. Res. 320: 207-215.

Latt, S. A., Allen, S. E., Loom, A. B., Carrano, E., Falke, D., Kram, E., Schneider, R., Schreck, R., Tice, B., White Field, B. and Wolff, S. 1981. Sister chromatid exchanges: A report of the gene-tox program. Mut. Res. 87: 17-62.

Lubran, M. M. 1989. Hematologic side effects of drugs. Ann. Clin. Lab. Sci. 19: 114-121.

Meissner, H. C. and Smith, A. L. 1979. The current status of chloramphenicol. Pediatrics 64: 348-356.

Nashwa, A. A. 2003. Cytogenetic studies on the effect of exposure to some Agrochemicals in goats. Ph.D. thesis. Fac. Vet. Med. Cairo Univrsity.

Plumb, D. C. 1999. Veterinary Drug Hand Book, 3rd ed. Iowa State University Press, Ames.

Paape, M. J., Miller, R. H. and Ziv, G. 1990. Effects of florfenicol, chloramphenicol and thiamphenicol on phagocytosis, chemiluminescence, and morphology of bovine polymorphnuclear neutrophil leukocytes. J. Dairy Sci. 73: 17341744 .

Robbana-Barnat, S., Decloitre, F., Frayssinet, C., Seigneurin, J. M., Toucas, L. and Lafarge-Frayssinet, C. 1997. Use of human lymphoblastoid cells to detect the toxic effect of chloramphenicol and its metabolites possibly involved in aplastic anemia in man. Drug Chem. Toxicol. 20: 239-253.

Sbrana, I., Caretto, S., Rainaldi, G. and Loprieno, N. 1991. Induction of chromosomal aberrations and SCE by chloramphenicol. Mutat. Res. 248: 145-153.

Schalm, O. W. 1986. Veterinary Haematology, 4th ed. Lea \& Febiger, Philadelphia.

Skolimowski, I. M., Knight, R. C. and Edwards, D. I. 1983. Molecular basis of chloramphenicol and thiamphenicol toxicity to DNA in vitro. J. Antimicrob. Chemother. 12: 535-542.

Summ, H. D., Draeger, E. and Wasielewski, E. 1976. On the inhibitory effect of chloramphenicol on mitochondrial protein synthesis as a possible cause of its selective toxic side effects. Arzneimittelforschung 26: 2832A.

Turton, J. A., Andrews, C. M., Havard, A. C. and Williams, T. C. 2002. Studies on the haemotoxicity of chloramphenicol succinate in the Dunkin Hartley guinea pig. Int. J. Exp. Pathol. 83: 225-238.

Watson, A. D. J. 1980. Further observations on chloramphenicol toxicosis in cats. Am. J. Vet. Res. 41: 293.

Wilson, W. R. and Cockerill, F. 1983. Tetracyclines, chloramphenicol, erythromycin and clindamycin. Mayo. Clin. Proc. 58: $92-98$. 